\title{
Pair production of magnetic monopoles and stable high-electric-charge objects in proton-proton and heavy-ion collisions
}

\author{
Wen-Yi Song* and Wendy Taylor ${ }^{\dagger}$ \\ Department of Physics and Astronomy, York University, Toronto, ON M3J 1P3 Canada
}

(Dated: February 28, 2022)

\begin{abstract}
We describe pair-production models of spin-0 and spin- $\frac{1}{2}$ magnetic monopoles and high-electriccharge objects in proton-proton and heavy-ion collisions, considering both the Drell-Yan and the photon-fusion processes. In particular, we extend the Drell-Yan production model of spin- $\frac{1}{2}$ highelectric-charge objects to include $Z^{0}$-boson exchange for proton-proton collisions. Furthermore, we explore spin- $\frac{1}{2}$ and, for the first time, spin-0 production in ultraperipheral heavy-ion collisions. With matrix element calculations and equivalent photon fluxes implemented in MADGRAPH5_aMC@NLO, we present leading-order production cross sections of these mechanisms in $\sqrt{s}=14 \mathrm{TeV}$ protonproton collisions and $\sqrt{s_{\mathrm{NN}}}=5.5 \mathrm{TeV}$ ultraperipheral lead-lead collisions at the LHC. While the mass range accessible in ultraperipheral lead-lead collisions is much lower than that in proton-proton collisions, we find that the theoretical production cross sections are significantly enhanced in the former for masses below $82 \mathrm{GeV}$.
\end{abstract}

Keywords: magnetic monopoles, high-electric-charge objects, pair production, heavy-ion collisions

\section{INTRODUCTION}

Maxwell's equations possess an electric-magnetic dual symmetry that is broken by the absence of magnetic charges. In 1931, Dirac showed that the existence of particles with magnetic charge was consistent with quantum mechanics [1. Since then, theories and models have been developed with different magnetic monopole solutions, as reviewed in [2] 7]. Unlike the composite monopoles in the 't Hooft-Polyakov model of grand unification theories 8, 9] or some extensions of the standard model 10 20, Dirac monopoles are point-like and structureless with unknown mass and spin. Interestingly, the observed quantization of electric charge is explained by Dirac's quantization condition [21, which dictates that a Dirac magnetic monopole carry a magnetic charge that is an integer multiple of the fundamental Dirac charge $g_{\mathrm{D}}$ :

$$
\frac{g_{\mathrm{D}} e}{\hbar c}=\frac{1}{2} \longrightarrow \frac{g_{\mathrm{D}}}{e}=\frac{1}{2 \alpha} \approx 68.5,
$$

in cgs Gaussian units, where $e$ is the elementary electric charge and $\alpha \approx 1 / 137$ is the fine-structure constant. This implies that the energy loss, or stopping power, of a Dirac monopole of magnetic charge $1 g_{\mathrm{D}}$ in matter is similar to that of an ion with electric charge $|z|=68.5$, where $z$ is in units of $e$ 22 24. Therefore, magnetic monopoles, as with high-electric-charge objects (HECOs), are highly ionising particles (HIPs). Another consequence of the Dirac quantization condition, which implies that electric and magnetic couplings cannot be simultaneously small due to their inverse correlation, is that monopoles cannot be handled with perturbation theory.

Monopole searches at colliders usually target stable Dirac magnetic monopoles and assume that magnetic charge is conserved [4, 7, 25, 26. Although the 't Hooft-Polyakov monopoles are too massive to be produced in colliders, numerous attempts have been undertaken to predict monopoles with masses accessible at colliders such as the LHC [1020]. There is no well-established theory of monopole production to date for direct cross section calculation due to their strong magnetic couplings. However, in order to obtain a mass limit that can be compared to existing experimental results, it is necessary to assume at least one model with an associated production cross section. Hence, the common approach is to formulate a magnetic-dual theory of Quantum Electrodynamics (QED) and consider possible benchmark scenarios. The benchmark model for monopole production has been the Drell-Yan (DY) mechanism but the photonfusion $(\mathrm{PF})$ mechanism [27 30] is now of interest, since at the current LHC energy scale its cross section in protonproton collisions is larger. Contrary to the inelastic Drell-Yan mechanism, photon fusion encompasses elastic and semi-elastic processes in addition to inelastic interactions.

To date, monopole and HECO searches at the LHC have been primarily conducted with proton-proton collisions with the general-purpose detector ATLAS as well as the dedicated MoEDAL experiment. The ATLAS collaboration

\footnotetext{
* wsong@yorku.ca

† taylorw@yorku.ca
} 
published the first LHC monopole search in 2012 based on $7 \mathrm{TeV}$ collision data collected in Run 1 [31] and has continuously proven to be particularly sensitive to monopoles of charge $1 g_{\mathrm{D}}$ and $2 g_{\mathrm{D}}$ and HECOs up to $|z|=100[32$ 34. The MoEDAL collaboration released its first monopole search result in 2016 using $8 \mathrm{TeV}$ data from Run 1 [35] and has been known for its capability to detect higher-charge monopoles and dyons [36 39.

The LHC not only collides protons but also heavy ions, which are of great interest due to the large electromagnetic fields associated with the high-charge ions moving at high velocities. Previously, monopole searches in heavy-ion collisions were conducted at the Brookhaven Alternating Gradient Synchrotron (AGS) using gold nuclei and at the CERN Super Proton Synchrotron (SPS) using lead nuclei with lead targets, placing lower mass limits of $3.3 \mathrm{GeV}$ and $8.1 \mathrm{GeV}$, respectively [40. Ultraperipheral collisions, where the ion-ion impact parameter exceeds the nucleus diameter, could produce magnetic monopoles via the photon-fusion mechanism. Furthermore, ultraperipheral collisions have a characteristic signature with low particle multiplicities, making them easy to select and analyze.

In this paper, we compare the different HIP pair-production mechanisms in proton-proton and heavy-ion collisions, with an emphasis on ultraperipheral lead-lead collisions. Section II identifies the interaction vertices for HIP production in Drell-Yan and photon-fusion mechanisms. Section III introduces heavy-ion collisions and explains ultraperipheral collisions as one collision type in lead-lead collisions. Section IV compares the parameters of collisions of various heavy ions to those of protons. Section $\mathrm{V}$ covers the implementation of the interaction vertices and the equivalent photon fluxes. Section VI presents the cross section results and representative kinematic distributions.

\section{PARTONIC MECHANISMS FOR HIP PAIR PRODUCTION}

The approximate duality between electric and magnetic charges in electromagnetism is the key to the development of a magnetic-dual theory of QED, which is mediated by photon exchange. QED has an underlying invariance under $U(1)$ gauge transformations, where the field of each charged particle picks up a phase proportional to its electric charge. This invariance fixes the coupling of the field to the photon and demands electric charge conservation at each vertex. The electromagnetic coupling to the photon for any electrically charged particle scales as the charge $|z|$. Hence, a minimal model of monopole interactions assumes an electromagnetic monopole-photon coupling that depends on the magnetic charge $N g_{\mathrm{D}}$ of the monopole, where $N$ is an integer. In the case of HECOs, it is completely natural to build additional interactions with a gauge invariance under $S U(2)$ transformations that govern the Standard-Model weak interactions, where the new gauge group gives rise to different matter-gauge couplings.

By constraining the possible interactions, the gauge symmetry allows us to write candidate Lagrangians from which we can compute the dynamics of the theory. We assume two spin states for the HIPs: spin- 0 and spin- $\frac{1}{2}$. For a given spin, the candidate $U(1)$ Lagrangian is the same for monopoles and HECOs except for the coupling, which depends on $N g_{\mathrm{D}}$ for monopoles and $|z|$ for HECOs. Hence, in the development below, we generalize monopoles and HECOs as HIPs with charge $g$. Working at leading order, we present two partonic HIP production mechanisms in proton-proton and heavy-ion collisions. The Drell-Yan mechanism is the process of a quark and an anti-quark annihilating to form a virtual photon (or a $Z^{0}$ boson in the case of HECOs), which then decays to a pair of HIPs. The photon-fusion mechanism is when two photons radiated from two colliding hadrons fuse to produce a pair of HIPs.

Invoking electric-magnetic duality, the charge and coupling of the magnetic monopole are often assumed to be velocity-dependent. This choice was based on the observed equivalence, in the small scattering angle limit, of the electron-monopole scattering differential cross section in [4] and the Rutherford scattering differential cross section, after substituting $\frac{g}{c}$ for $\frac{e}{v}\left[\underline{4}\right.$. The resulting magnetic charge $g \frac{v}{c} \equiv g \beta$, not only appears in the description of monopole energy loss, but also when considering monopole pair production via the Drell-Yan and photon-fusion mechanisms [27[30, 35] 38, 42. We choose to follow the approach of a minimal model for Dirac monopoles where all HIP charges and couplings are assumed to be velocity-independent. Doing so leads to slightly different production cross sections and kinematic features. While the velocity dependence suppresses production of monopoles with lower velocities, a velocity-independent monopole coupling does not, thereby giving rise to softer kinematic distributions and higher production cross sections.

\section{A. Spin-0 interaction}

The production of spin-0 HIPs in the magnetic-dual theory of scalar QED is described by the candidate Lagrangian

$$
\mathcal{L}=-\frac{1}{4} F^{\mu \nu} F_{\mu \nu}+\left(D^{\mu} \phi\right)^{\dagger}\left(D_{\mu} \phi\right)-m^{2} \phi^{\dagger} \phi
$$


where the covariant derivative

$$
D_{\mu}=\partial_{\mu}+i g A_{\mu}
$$

couples the spin-0 HIP field, $\phi$, to the photon field, $A_{\mu}$, whose field strength is $F_{\mu \nu}=\partial_{\mu} A_{\nu}-\partial_{\nu} A_{\mu}$. As a result, the covariant derivative component of 2 gives rise to the HIP interactions

$$
\mathcal{L}_{\text {int }}=\mathcal{L}_{3 \mathrm{pt}}+\mathcal{L}_{4 \mathrm{pt}},
$$

whose components

$$
\mathcal{L}_{3 \mathrm{pt}}=-i g A_{\mu}\left(\phi^{\dagger} \partial^{\mu} \phi-\phi \partial^{\mu} \phi^{\dagger}\right)
$$

and

$$
\mathcal{L}_{4 \mathrm{pt}}=g^{2} A^{\mu} \phi^{\dagger} A_{\mu} \phi
$$

dictate the vertices shown in figure 1

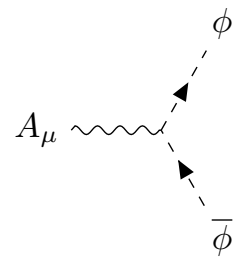

(a) Three-point vertex

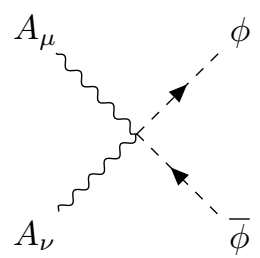

(b) Four-point vertex

FIG. 1. Two vertices given by scalar HIP-photon interactions.

While the Drell-Yan mechanism for scalar HIP pair production, shown in figure 2, realizes only the three-point vertex, the photon-fusion process, shown in figure 3, has contributions from both the three-point and four-point vertices.

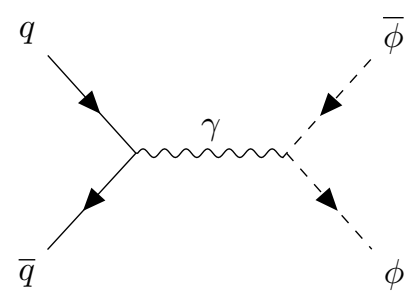

FIG. 2. Feynman diagram of the photon-exchange Drell-Yan mechanism to produce a pair of scalar HIPs.

The interaction between the spin-0 HECOs and the $Z^{0}$ boson can be derived through the gauge invariance principle. Spin-0 HECOs couple to the $Z^{0}$ boson via tri-linear and quadra-linear interactions, therefore, they can only interact with a pair of $Z^{0}$ bosons assuming Standard-Model interactions, as shown in figure 4 . That is, the spin-0 HECOs cannot be produced via the $Z^{0}$-exchange-mediated Drell-Yan mechanism.

\section{B. Spin- $\frac{1}{2}$ interaction}

Assuming HIPs are Dirac fermions, the basic Lagrangian for a spin- $\frac{1}{2}$ field $\psi$ is

$$
\mathcal{L}=-\frac{1}{4} F^{\mu \nu} F_{\mu \nu}+\bar{\psi} i \gamma^{\mu} D_{\mu} \psi-m \bar{\psi} \psi
$$

with a different covariant derivative component of $\bar{\psi} i \gamma^{\mu} D_{\mu} \psi$ compared to that of the spin-0 scenario. This distinction gives rise to a different HIP-photon interaction term

$$
\mathcal{L}_{\text {int }}=-g \bar{\psi} \gamma^{\mu} A_{\mu} \psi
$$




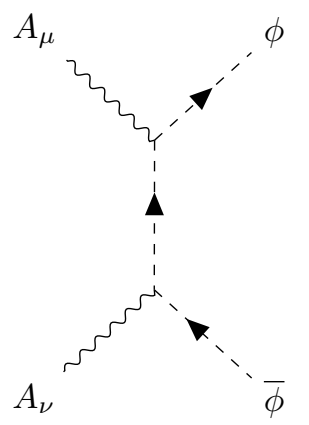

(a) $t$-channel

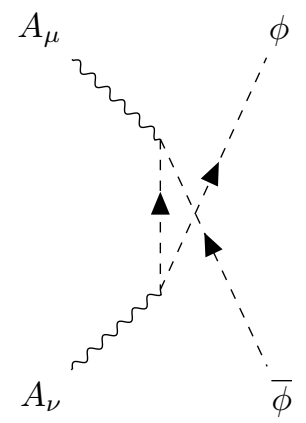

(b) u-channel

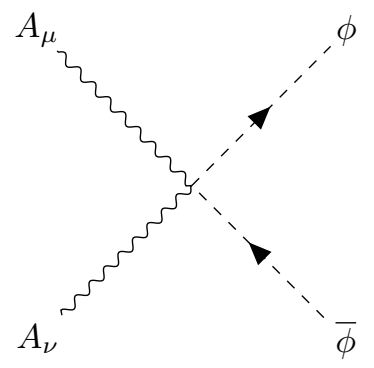

(c) Four-point interaction

FIG. 3. Feynman diagrams of photon fusion to produce a pair of scalar HIPs.
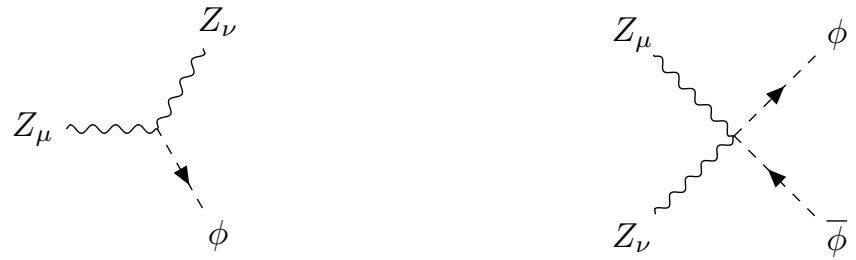

FIG. 4. Feynman diagrams of tri-linear (left) and quadra-linear (right) interactions of spin-0 HECOs with $Z^{0}$ bosons.

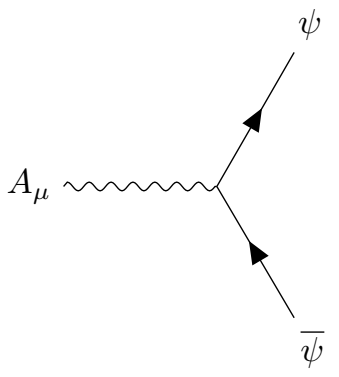

FIG. 5. Three-point vertex for the interaction of spin- $\frac{1}{2}$ HIPs with a photon.

which corresponds to the three-point vertex of spin- $\frac{1}{2}$ HIPs in figure 5 . With this vertex, the photon-mediated DrellYan mechanism in figure 6 was the first production process considered for HIP production at the LHC. In addition, this three-point vertex contributes to the photon-fusion production process in figure 7 , where two such vertices are involved for a given production channel.

While the magnetically charged monopoles are only allowed to interact via the electromagnetic neutral current, the electrically charged HECOs, like many other particles with exotic electric charges [43, 44], can also interact via the weak neutral current, as we assume they transform under the $S U(2)$ symmetry of the weak interactions. To build such a model, a local $S U(2)$ gauge invariance is imposed to generate a coupling to $Z^{0}$ bosons via a covariant derivative 


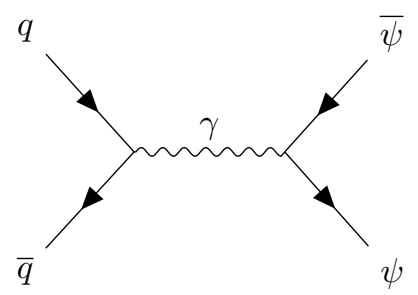

FIG. 6. Feynman diagram of the photon-exchange Drell-Yan mechanism to produce a pair of fermionic HIPs.
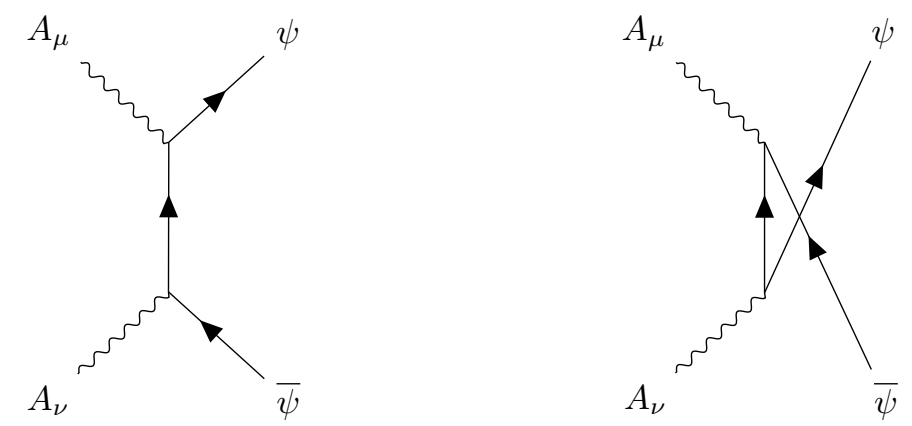

FIG. 7. Feynman diagrams of photon fusion to produce a pair of fermionic HIPs.

analogous to 3 . This interaction is given by

$$
\mathcal{L}_{\text {int }}^{\prime}=-\frac{e}{2 \sin \theta_{\mathrm{W}} \cos \theta_{\mathrm{W}}} \bar{\psi} \gamma^{\mu}\left(c_{\mathrm{L}} P_{\mathrm{L}}+c_{\mathrm{R}} P_{\mathrm{R}}\right) \psi Z_{\mu},
$$

where $e$ is the electromagnetic coupling, $\theta_{\mathrm{W}}$ is the weak mixing angle, and $P_{\mathrm{L}}$ and $P_{\mathrm{R}}$ are the chiral projectors. Unlike the HIP-photon interaction in 5 , this Lagrangian has a chirality dependence parametrized by two couplings that are generally not identical:

$$
c_{\mathrm{L}}=t_{3}-|z| \sin ^{2} \theta_{\mathrm{W}} \quad \text { and } \quad c_{\mathrm{R}}=-|z| \sin ^{2} \theta_{\mathrm{W}}
$$

where $t_{3}$ is the weak isospin of the HIPs. The assumption that HECOs transform as $S U(2)$ singlets implies that $t_{3}=0$ for HECOs. The interaction Lagrangian in 6 is associated with the three-point vertex in figure 8, which drives the Drell-Yan production of spin- $\frac{1}{2}$ HECOs via $Z^{0}$-boson exchange, as in figure 9 .

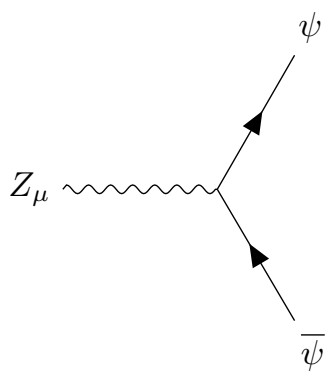

FIG. 8. Three-point vertex for the interaction of spin- $\frac{1}{2}$ HECOs with a $Z^{0}$ boson.

\section{ULTRAPERIPHERAL HEAVY-ION COLLISIONS}

Heavy-ion collisions have many distinctive properties that make them a unique environment for studying a wide range of interactions, including the production of magnetic monopoles. The impact parameter of the colliding ions 


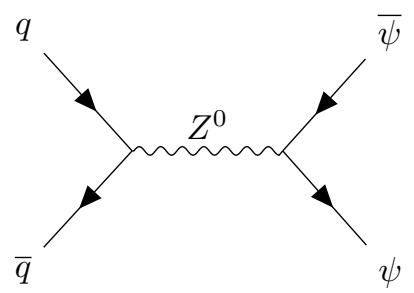

FIG. 9. Feynman diagram of the $Z^{0}$-exchange Drell-Yan mechanism to produce a pair of fermionic HECOs.

characterizes the effective interaction in heavy-ion collisions. When the ions interact with impact parameters less than or equal to twice the nuclear radii, the strong force governs the interaction between them. These are known as central collisions and their extraordinarily high temperatures can produce a quark-gluon plasma. However, when the two ions pass each other with an impact parameter much larger than twice their radii, strong interactions between the nucleons are no longer possible. Nevertheless, even at large impact parameters electromagnetic processes between the ions can occur. In these so-called ultraperipheral collisions (UPC), photons radiated from the ions interact via the photon-fusion process while the ions remain intact.

The prospect of monopole production in heavy-ion collisons has a long history of interest. Initially, it was envisioned to occur via thermal processes of central collisions where the massive monopoles would couple to the quark-gluon plasma [45] via photons. Later, monopole production was postulated in ultraperipheral elastic photon-fusion processes in electron-electron, proton-proton and lead-lead collisions [42, motivated by the enhanced cross sections due to the high electric charges of the heavy ions over protons. Recently, monopole production has been considered again in ultraperipheral heavy-ion collisions [46 52], where the strong magnetic field from the ultrarelativistic high-charge ions passing each other may produce monopoles via the magnetic-dual mechanism of the thermal Schwinger process [53. This production mechanism has been considered in an experimental search for the first time by the MoEDAL collaboration [54. The present study first focuses on the electromagnetic radiation emitted by the ultrarelativistic ions, as in [42, then continues to formulate a model of monopole and HECO production in (the magnetic dual of) QED, assuming the effective interaction is photon fusion.

\section{A. Electromagnetic radiation emitted by relativistic heavy ions}

We review the equivalent photon method, which describes the electromagnetic radiation emitted in heavy-ion collisions. The original formalism, proposed by Enrico Fermi in 1924 [55], treated the time-varying electromagnetic field as a flux of virtual photons. Later, it was adapted by Weizsäcker and Williams [56, 57, for the photon fluxes of relativistic ions.

We consider a particle with electric charge $Z$ as approximately a point particle. At ultrarelativistic velocities, it produces electric and magnetic fields $(\mathbf{E}$ and $\mathbf{B})$ such that the total energy they carry propagates as a flux of equivalent photons with its direction and magnitude dictated by $\mathbf{E} \times \mathbf{B}$. The resulting photon density spectrum $d n_{\gamma}$ possesses a $Z^{2}$ dependence and can be defined for a given pair of energy $\omega$ and impact parameter $b$ in the plane transverse to its direction of motion. The number density over the entire transverse plane is obtained by integrating over all possible impact parameters $b$. Therefore, the number density per energy is

$$
\frac{d n_{\gamma}}{d \omega}(\omega)=2 \pi \int_{b_{\min }}^{\infty} \frac{d n_{\gamma}}{d \omega}(\omega, b) b d b
$$

Defined with respect to one particle, this impact parameter, denoted as $b$ hereafter, should be distinguished from the collision impact parameter that categorizes the collision type. If the two colliding particles have the same size, the particle impact parameter $b$ is half of the collision impact parameter on average.

Now we consider the scenario of two charged particles moving towards each other at ultrarelativistic velocities. In an ultraperipheral collision, the nuclei remain intact and the interactions take place at a finite distance, which in turn restricts the kinematics of the virtual photons due to the uncertainty principle. In consequence, the emitted photon's wavelength given by the impact parameter $b$ has a lower bound of the nuclear radius $R_{A}$, which corresponds to a maximal transverse momentum of $\hbar / R_{A}$. Since the photon's off-shell mass is suppressed by the impact parameter, its virtuality decreases with the size of the charged particle. In the longitudinal direction, the photon's momentum is boosted by a Lorentz factor, reaching an effective maximum of $\gamma \hbar / R_{A}$. It follows from the $1 / R_{A}$ dependence that the photon energy bound is more stringent in heavy-ion collisions than in proton-proton collisions. 


\section{PROTON-PROTON AND HEAVY-ION COLLISIONS AT THE LHC}

Theories describing the production of magnetic monopoles and HECOs provide little guidance as to their masses. However, when it comes to collider searches, the accessible mass range is bounded by the collision energy. At the LHC collision energy, it is unlikely to produce HIPs more massive than $4000 \mathrm{GeV}$ via either the Drell-Yan or the photonfusion mechanism, given that the interacting partons only carry a fraction $x$ of the beam energy. In ultraperipheral heavy-ion collisions, the even smaller accessible mass range is restricted by the maximum energy of the emitted photon, $\gamma \hbar / R_{A}$, which is $82 \mathrm{GeV}$ for lead-lead collisions.

Table I compares the parameters of ultraperipheral collisions between different particles at the LHC design beam energy, where the ions are fully stripped with no remaining electrons. The proton-proton collision center-of-mass energy $\sqrt{s}$ is equal to the sum of the proton beam energies, i.e., $13 \mathrm{TeV}$ in Run 2 at the LHC. The heavy-ion centerof-mass energy $\sqrt{s_{\mathrm{NN}}}$ is determined by the energy carried by the protons in the colliding beams, since only the protons in the ions are accelerated. As a consequence, given the same beam energy, $\sqrt{s_{\mathrm{NN}}}$ for heavy-ion collisions is lower than $\sqrt{s}$ for proton-proton collisions. Given the $1 / R_{A}$ dependence of the maximal transverse momenta $p_{\mathrm{T}}^{\max }$ for the different collision systems, the $p_{\mathrm{T}}$ profile of the photons is highly suppressed in a heavy-ion collision compared to a proton-proton collision.

TABLE I. Parameters in different ultraperipheral collision systems with the same beam energy $E_{\text {beam }}$ : nucleon-nucleon centerof-mass energy $\sqrt{s_{\mathrm{NN}}}$, Lorentz factor $\gamma$, nuclear radius $R_{A}$, charge-induced photon luminosity enhancement $Z^{4}$, charge-to-mass ratio $Z / A$, maximal transverse momentum $p_{\mathrm{T}}^{\max }$, maximal longitudinal momentum $p_{\mathrm{L}}^{\max }$ and maximal photon-photon centerof-mass energy $\sqrt{s_{\gamma \gamma}^{\max }}$.

\begin{tabular}{cccccccccc}
\hline \hline & $\begin{array}{c}E_{\text {beam }} \\
{[\mathrm{TeV}]}\end{array}$ & $\begin{array}{c}\sqrt{s_{\mathrm{NN}}} \\
{[\mathrm{TeV}]}\end{array}$ & $\gamma$ & $R_{A}[\mathrm{fm}]$ & $Z^{4}$ & $Z / A$ & $p_{\mathrm{T}}^{\max }[\mathrm{MeV}] p_{\mathrm{L}}^{\max }[\mathrm{GeV}]$ & $\begin{array}{c}\sqrt{s_{\gamma \gamma}^{\max }} \\
{[\mathrm{GeV}]}\end{array}$ \\
\hline $\mathrm{p}$ & 7.0 & 14 & $7.5 \times 10^{3}$ & $0.8[58]$ & 1.0 & 1.0 & 237 & $1.8 \times 10^{3}$ & $3.6 \times 10^{3}$ \\
${ }_{8}^{16} \mathrm{O}$ & 7.0 & 7.0 & $3.8 \times 10^{3}$ & 3.0 & $4.1 \times 10^{3}$ & 0.50 & 65 & 245 & 490 \\
${ }_{54}^{129} \mathrm{Xe}$ & 7.0 & 5.9 & $3.1 \times 10^{3}$ & 6.1 & $8.5 \times 10^{6}$ & 0.42 & 32 & 102 & 204 \\
${ }_{82}^{208} \mathrm{~Pb}$ & 7.0 & 5.5 & $3.0 \times 10^{3}$ & 7.1 & $4.5 \times 10^{7}$ & 0.39 & 28 & 82 & 164 \\
\hline \hline
\end{tabular}

The LHC has a long-standing interest in collisions between lighter ions, which have an advantage in ultraperipheral collisions. In Run 2, the LHC delivered xenon-xenon collisions in addition to lead-lead collisions. Different collision systems have different ratios of atomic number $Z$ and mass number $A$. Hence, $\sqrt{s_{\mathrm{NN}}}$ is higher for an ion with a higher ratio of atomic number $Z$ to mass number $A$, for a fixed beam energy. Heavy nuclei with high $Z$ values have significantly stronger electrostatic interactions between the protons extending beyond their sizes. Hence, higher fractions of neutrons are necessary for the short-range strong interaction between nucleons to keep the ion stable. In other words, light ions such as oxygen have higher $Z / A$ values than the commonly considered xenon and lead ions. The energy fraction carried by the protons will increase and result in higher photon-photon collisions energies. The LHC foresees the addition of oxygen-oxygen collisions to its heavy-ion collision program in Run 3.

\section{MADGRAPH IMPLEMENTATION AND RESULTS}

The HIP production model is built within an event generator called MADGRAPH5_aMC@NLO [59]. The model itself defines particles and their interactions that are passed to the MADGRAPH5_aMC@NLO generator for every user-specified process of interest. Next, MADGRAPH5_aMC@NLO searches for all interaction vertices in the specified model that accommodate the incoming and outgoing particles the user provides to generate a valid hard process through a set of Feynman diagrams. It then calculates the matrix element of the hard process from the Feynman diagrams via perturbation theory to a finite order. It outputs the production cross section as an integral of the matrix element over the initial momenta of the incoming particles and the phase space of the outgoing particles. In hadron collisions, the probability of finding an incoming particle or a parton at a given momentum follows the parton distribution function (PDF). The MADGRAPH5_aMC@NLO generator provides different PDF options via the LHAPDF library. 


\section{A. Couplings}

Every HIP interaction vertex described in section II is characterized by a coupling constant that is proportional to the HIP charge. MADGRAPH5_aMC@NLO uses Heaviside-Lorentz units such that one electron charge is $\sqrt{4 \pi}$ times the electron charge in the Gaussian units. Thus the fine structure constant is written as $\alpha=\frac{e^{2}}{4 \pi}$, where the electron coupling $e$ in Heaviside-Lorentz units is

$$
e=2 \sqrt{\alpha \pi}
$$

As a result, the HECO couplings are integer multiples of the electron coupling, while the monopole couplings are integer multiples of $g_{\mathrm{D}}$, obtained by rearranging 1 and substituting 9

$$
g_{\mathrm{D}}=\frac{2 \pi}{\sqrt{4 \pi \alpha}}=\sqrt{\frac{\pi}{\alpha}} .
$$

\section{B. Spin-0 and spin- $\frac{1}{2}$ photon-HIP vertices}

The spin-0 HIP-photon interaction features the two vertices in figure 1, where the three-point vertex is needed for both the Drell-Yan and the photon-fusion mechanisms while the four-point vertex is only relevant for photon fusion. On the contrary, the spin- $\frac{1}{2}$ HIP's interactions with a photon only feature the three-point vertex, which is responsible for both the Drell-Yan induced and the photon-fusion induced HIPs. For a given spin scenario, the two different partonic mechanisms can be validated through the validation of the same vertices. For each vertex, it suffices to validate one interaction that involves it. Since the photon-fusion mechanism utilizes all three vertices, including the additional spin-0 four-point vertex, a successfully constructed photon-fusion mechanism guarantees the validity of its Drell-Yan counterpart. Hence, we validate the photon-HIP vertices in photon-fusion interactions.

Since the cross section depends explicitly on the center-of-mass energy, it is ideal to work with collisions with a fixed center-of-mass energy. However, the momenta of the photons emitted by two colliding hadrons follow a nontrivial distribution such that the effective photon collisions have no fixed center-of-mass energy. For the ease of the theoretical calculations, the photon-fusion process is modeled in MADGRAPH5_aMC@NLO by colliding bare photons, i.e., photons that are not emitted by hadrons. In this case, the initial momenta of the photons are automatically set to a constant value given the fixed collision energy. Cross sections are obtained for the photon collisions generated with MADGRAPH5_aMC@NLO and calculated analytically to validate the vertex implementation. Given all the vertices discussed in section II], the production cross sections at tree level are computed to be

$$
\begin{gathered}
\sigma(\gamma \gamma \rightarrow \phi \bar{\phi})=\frac{g^{4}}{64 \pi}\left[\frac{2 \beta}{E^{2}}+\frac{2 \beta m^{4}}{E^{6}}+\frac{E^{2}\left(1-\beta^{2}\right)^{2}-2 m^{2}}{E^{4}} \ln \left(\frac{1+\beta}{1-\beta}\right)\right], \quad \text { for spin-0 HIPs, and } \\
\sigma(\gamma \gamma \rightarrow \psi \bar{\psi})=\frac{g^{4}}{32 \pi E^{2}}\left[2 \beta^{3}-4 \beta+\frac{2 E^{4}+2 E^{2} m^{2}-m^{4}}{E^{4}} \ln \left(\frac{1+\beta}{1-\beta}\right)\right], \quad \text { for spin- } \frac{1}{2} \text { HIPs, }
\end{gathered}
$$

where $E$ is the energy of each photon, $g$ stands for the coupling strength, $\beta$ is the normalized velocity and $m$ is the rest mass for each HIP.

The photon-fusion cross sections are generated by the MADGRAPH5_aMC@NLO model with two colliding photons, each carrying a fixed energy of $7 \mathrm{TeV}$. Clearly, the $14 \mathrm{TeV}$ center-of-mass energy for the two-photon system is beyond the reach of the LHC. However, for validation purposes this energy can be arbitrarily chosen, as long as it is greater than the total mass of the final-state particles. The MADGRAPH5_aMC@NLO model calculation is validated against theory computed from 11 and 12 for $1 g_{\mathrm{D}}$ monopoles in table II and for $|z|=60$ HECOs (for their approximate equivalence to $1 g_{\mathrm{D}}$ monopoles dictated by 1 in table III.

\section{Spin- $\frac{1}{2}$ Drell-Yan $Z^{0}$-exchange vertex for HECOs}

The implementation of the $Z^{0}$ boson decay to a pair of spin- $\frac{1}{2}$ HECOs is similar to that of the decay of a $Z^{0}$ boson to an electron-positron pair, apart from the necessary modifications to represent the zero weak isospin and $|z|>1$ electric charges of the HECOs. This vertex is further examined with cross-section calculations in MADGRAPH5_aMC@NLO 
TABLE II. Comparison of cross sections obtained from the model and calculated analytically for $|g|=1 g_{\mathrm{D}}$ monopoles of various mass-spin combinations produced via photon fusion (PF) at a fixed center-of-mass energy of $14 \mathrm{TeV}$.

\begin{tabular}{ccccccc}
\hline \hline $\begin{array}{c}\text { Mass } \\
(\mathrm{GeV})\end{array}$ & \multicolumn{2}{c}{ Spin-0 } & $1 g_{\mathrm{D}}$ monopole PF $\sigma[\mathrm{pb}]$ & \multicolumn{3}{c}{ Spin- $\frac{1}{2} 1 g_{\mathrm{D}}$ monopole PF $\sigma[\mathrm{pb}]$} \\
& Mheory & Ratio $(\mathrm{M} / \mathrm{T})$ & Model & Theory & Ratio $(\mathrm{M} / \mathrm{T})$ \\
\hline 10 & $1.370 \times 10^{4}$ & $1.370 \times 10^{4}$ & 1.000 & $3.696 \times 10^{5}$ & $3.685 \times 10^{5}$ & 1.000 \\
50 & $1.369 \times 10^{4}$ & $1.369 \times 10^{4}$ & 1.000 & $2.815 \times 10^{5}$ & $2.814 \times 10^{5}$ & 1.000 \\
100 & $1.367 \times 10^{4}$ & $1.367 \times 10^{4}$ & 1.000 & $2.434 \times 10^{5}$ & $2.434 \times 10^{5}$ & 1.000 \\
500 & $1.328 \times 10^{4}$ & $1.327 \times 10^{4}$ & 1.001 & $1.560 \times 10^{5}$ & $1.560 \times 10^{5}$ & 1.000 \\
1000 & $1.238 \times 10^{4}$ & $1.238 \times 10^{4}$ & 1.000 & $1.196 \times 10^{5}$ & $1.196 \times 10^{5}$ & 1.000 \\
3000 & 7835 & 7836 & 1.000 & $6.600 \times 10^{4}$ & $6.602 \times 10^{4}$ & 1.000 \\
5000 & 5455 & 5450 & 1.001 & $3.663 \times 10^{4}$ & $3.660 \times 10^{4}$ & 1.001 \\
\hline \hline
\end{tabular}

TABLE III. Comparison of cross sections obtained from the model and calculated analytically for $|z|=60$ HECOs of various mass-spin combinations produced via photon fusion $(\mathrm{PF})$ at a fixed center-of-mass energy of $14 \mathrm{TeV}$.

\begin{tabular}{|c|c|c|c|c|c|c|}
\hline \multirow{2}{*}{$\begin{array}{c}\text { Mass } \\
(\mathrm{GeV})\end{array}$} & \multicolumn{3}{|c|}{ Spin-0 $|z|=60$ HECO PF $\sigma[\mathrm{pb}]$} & \multicolumn{3}{|c|}{ Spin- $-\frac{1}{2}|z|=60$ HECO PF $\sigma[\mathrm{pb}]$} \\
\hline & Model & Theory & Ratio $(\mathrm{M} / \mathrm{T})$ & Model & Theory & Ratio $(\mathrm{M} / \mathrm{T})$ \\
\hline 10 & 9215 & 9213 & 1.000 & $2.486 \times 10^{5}$ & $2.486 \times 10^{5}$ & 1.000 \\
\hline 50 & 9211 & 9208 & 1.000 & $1.894 \times 10^{5}$ & $1.892 \times 10^{5}$ & 1.001 \\
\hline 100 & 9196 & 9196 & 1.000 & $1.637 \times 10^{5}$ & $1.637 \times 10^{5}$ & 1.000 \\
\hline 500 & 8930 & 8924 & 1.001 & $1.049 \times 10^{5}$ & $1.049 \times 10^{5}$ & 1.000 \\
\hline 1000 & 8328 & 8325 & 1.000 & $8.046 \times 10^{4}$ & $8.042 \times 10^{4}$ & 1.000 \\
\hline 3000 & 5270 & 5271 & 1.000 & $4.439 \times 10^{4}$ & $4.441 \times 10^{4}$ & 1.000 \\
\hline 5000 & 3669 & 3666 & 1.001 & $2.464 \times 10^{4}$ & $2.462 \times 10^{4}$ & 1.001 \\
\hline
\end{tabular}

itself to look for the expected charge dependence. The cross section $\sigma$ goes as the absolute square of the matrix element $\mathcal{M}$, given by the interaction Lagrangian in 6 , which scales as the couplings $c_{\mathrm{L} / \mathrm{R}}$ in 7

$$
\sigma \propto|\mathcal{M}|^{2} \propto\left|c_{\mathrm{L}}+c_{\mathrm{R}}\right|^{2} \propto|z|^{2}
$$

That is, the cross section of the $Z^{0}$-exchange mode grows as $|z|^{2}$, just as in the photon exchange mode. Cross sections for both modes are listed for mass $1000 \mathrm{GeV}$ HECOs in table IV for a range of charges: $|z|=2$ and 7 as considered in multi-charged particle searches [60 64, along with five HECO charges centered at $|z|=60$. The two exchange modes destructively interfere as a result of the relative sign between the two interactions parametrized in 5 and 6 , hence, the total cross section is smaller than the photon-exchange cross section alone.

TABLE IV. Cross sections for $1000 \mathrm{GeV}$ spin- $\frac{1}{2}$ HECOs with varying charges in different Drell-Yan production modes.

\begin{tabular}{cccc}
\hline \hline $\begin{array}{c}\text { Charge } \\
|z|\end{array}$ & \multicolumn{2}{c}{ Drell-Yan $\sigma$ for $1000 \mathrm{GeV}$ Spin- $\frac{1}{2}$ HECOs $[\mathrm{pb}]$} \\
& $\gamma$ exchange & $Z^{0}$ exchange & $\gamma / Z^{0}$ exchange \\
\hline 2 & $2.191 \times 10^{-4}$ & $8.903 \times 10^{-5}$ & $1.968 \times 10^{-4}$ \\
7 & $2.684 \times 10^{-3}$ & $1.091 \times 10^{-3}$ & $2.411 \times 10^{-3}$ \\
20 & 0.02191 & $8.903 \times 10^{-3}$ & 0.01968 \\
40 & 0.08765 & 0.03561 & 0.07871 \\
60 & 0.1972 & 0.08013 & 0.1771 \\
80 & 0.3506 & 0.1425 & 0.3148 \\
100 & 0.5478 & 0.2226 & 0.4919 \\
\hline \hline
\end{tabular}




\section{Parton distribution functions}

The total cross section $\sigma_{A B}$ of an arbitrary A-B collision can be expressed as the hard parton-level sub-process cross section $\hat{\sigma}_{a b}$ reweighted with the appropriate parton distribution functions (PDF) $f_{a / A}$ and $f_{b / B}$ for the initial state momentum fractions $x_{a}$ and $x_{b}$ :

$$
\sigma_{A B}=\int d x_{a} d x_{b} f_{a / A}\left(x_{a}\right) f_{b / B}\left(x_{b}\right) \hat{\sigma}_{a b} .
$$

In proton-proton collisions, $\mathrm{A}$ and $\mathrm{B}$ represent the incoming protons and the initial momentum fractions $x_{i}(i=a, b)$ are evaluated with respect to the protons. If the partonic mechanisms consider different initial states, as for Drell-Yan and photon fusion, then the parton distribution functions $f_{a / A}$ and $f_{b / B}$ will be different. In MADGRAPH5_aMC@NLO, the NNPDF23LO_qed PDF [65] was used for Drell-Yan production while the LUXqed17 PDF [66, 67] was used for photon fusion, due to the intrinsic uncertainties of the PDFs. The quark and gluon PDFs are relatively well constrained, with uncertainties typically of order $5 \%$ 68, while the photon PDF is weakly constrained due to the other processes present in the LHC data. With uncertainties up to 50\% [69, the photon PDFs ultimately lead to large uncertainties in the predicted cross sections of the photon fusion process. This disadvantage is specifically targeted in the calculation of the LUXqed17 PDF, where electron-proton scattering data was collected to determine the photon PDF. The uncertainties in this measurement are significantly reduced to 1-2\% 66] for a wide momentum range, as the momentum transfers in the scattering processes are carried by photons emitted from the protons.

\section{E. Equivalent photon fluxes for ultraperipheral heavy-ion collisions}

In heavy-ion ultraperipheral collisions, the total cross section for HIP production factorizes into the product of the parton-level cross section convoluted with the equivalent photon spectra from the two colliding ion beams. The parton-level interaction is the same photon fusion as discussed for proton-proton collisions, while the photon spectrum is uniquely parametrized for a given nuclear charge $Z$ that follows 8 in the Weizsäcker-Williams approach [70,

$$
f_{\gamma}(x)=\frac{Z^{2} \alpha}{\pi} \frac{1}{x}\left[2 x_{i} K_{0}\left(x_{i}\right) K_{1}\left(x_{i}\right)-x_{i}^{2}\left(K_{1}^{2}\left(x_{i}\right)-K_{0}^{2}\left(x_{i}\right)\right],\right.
$$

where $x_{i}=x m_{N} b_{\min }$ builds in the Lorentz boost effect through the atomic mass $m_{N}$ and excludes hadronic interactions via the minimal impact parameter requirement $b_{\min }=R_{A}$, while $K_{0,1}$ are the modified Bessel functions of the second kind of zero and first order that encode longitudinal and transverse polarizations of the spectrum. In principle, there is no constraint on the intactness of the nuclei for an ultraperipheral collision to take place, as long as there is no hadronic component in the same collision event. However, in a typical calculation, a cut of the nuclear radius is applied to the impact parameter to ensure the absence of hadronic activity. The nuclear charge dependence of the photon spectrum scales as $Z^{2}$, enhancing the cross section in heavy-ion collisions by $Z^{4}$ compared to photon fusion in proton-proton collisions. The photons are transversely polarized for relativistic ions, as parametrized by the dominance of $K_{1}$ over $K_{0}$.

The implemented $K_{0,1}$ factors are polynomial approximations of the true $K_{0,1}$ functions, with a precision on the order of $10^{-7}$ [71]. For a lead ion with 82 protons and 126 neutrons, the atomic mass $m_{N}$ is set to 208 times the proton mass. The radius $R_{A}$ is set to $6.64 \mathrm{fm}$.

\section{RESULTS AND CONCLUSIONS}

We compared the cross sections and kinematics for spin-0 and spin- $\frac{1}{2}$ production of monopoles and HECOs via photon fusion in ultraperipheral collisions to those for the Drell-Yan and photon-fusion mechanisms in proton-proton collisions for the LHC design collision energy of $\sqrt{s}=14 \mathrm{TeV}$. Since the photons emitted by the lead ion beams are kinematically bounded by $82 \mathrm{GeV}$ at the lead-lead collision energy of $\sqrt{s_{\mathrm{NN}}}=5.5 \mathrm{TeV}$, the HIP mass range accessible in ultraperipheral collisions is much lower than that of proton-proton collisions. Hence, we examined $1 g_{\mathrm{D}}$ monopoles and $|z|=60$ HECOs with masses below $82 \mathrm{GeV}$. The implemented photon spectrum acts as the photon PDF for ultraperipheral HIP production.

The production cross sections of spin- $01 g_{\mathrm{D}}$ monopoles and $|z|=60$ HECOs are depicted in figure 10 where three different collision modes are presented. The lead-lead ultraperipheral production is compared to proton-proton production via the Drell-Yan and the photon-fusion mechanisms. The ultraperipheral production dominates the 
proton-proton production mechanisms at the considered mass range. As far as proton-proton collisions are concerned, the photon-fusion mechanism is dominant at $\sqrt{s}=14 \mathrm{TeV}$.

The ultraperipheral production cross sections of $1 g_{\mathrm{D}}$ monopoles and $|z|=60$ HECOs are shown in figure 11 . where the spin- $\frac{1}{2}$ HIPs have larger cross sections than the spin-0 HIPs.

The kinematic distributions for ultraperipheral production of $1 g_{\mathrm{D}}$ monopoles with mass $40 \mathrm{GeV}$ are presented in figure 12. It should be noted that the kinematics are independent of HIP charge. The transverse momentum distribution is softer for spin-0 HIPs than for spin- $\frac{1}{2}$ HIPs, as a result of the four-point vertex in the spin-0 photonfusion mechanism. The pseudorapidity distributions are very similar in the two spin scenarios.

Finally, we compare the cross sections for the Drell-Yan and photon-fusion mechanisms in proton-proton collisions. The typical masses considered in the LHC proton-proton collisions are on the order of 1 TeV. As shown in figure 13 . the cross sections are higher for photon fusion than for Drell Yan production. As was the case in the ultraperipheral collisions, the cross sections for spin- $\frac{1}{2}$ HIPs are larger than those for spin-0 HIPs.
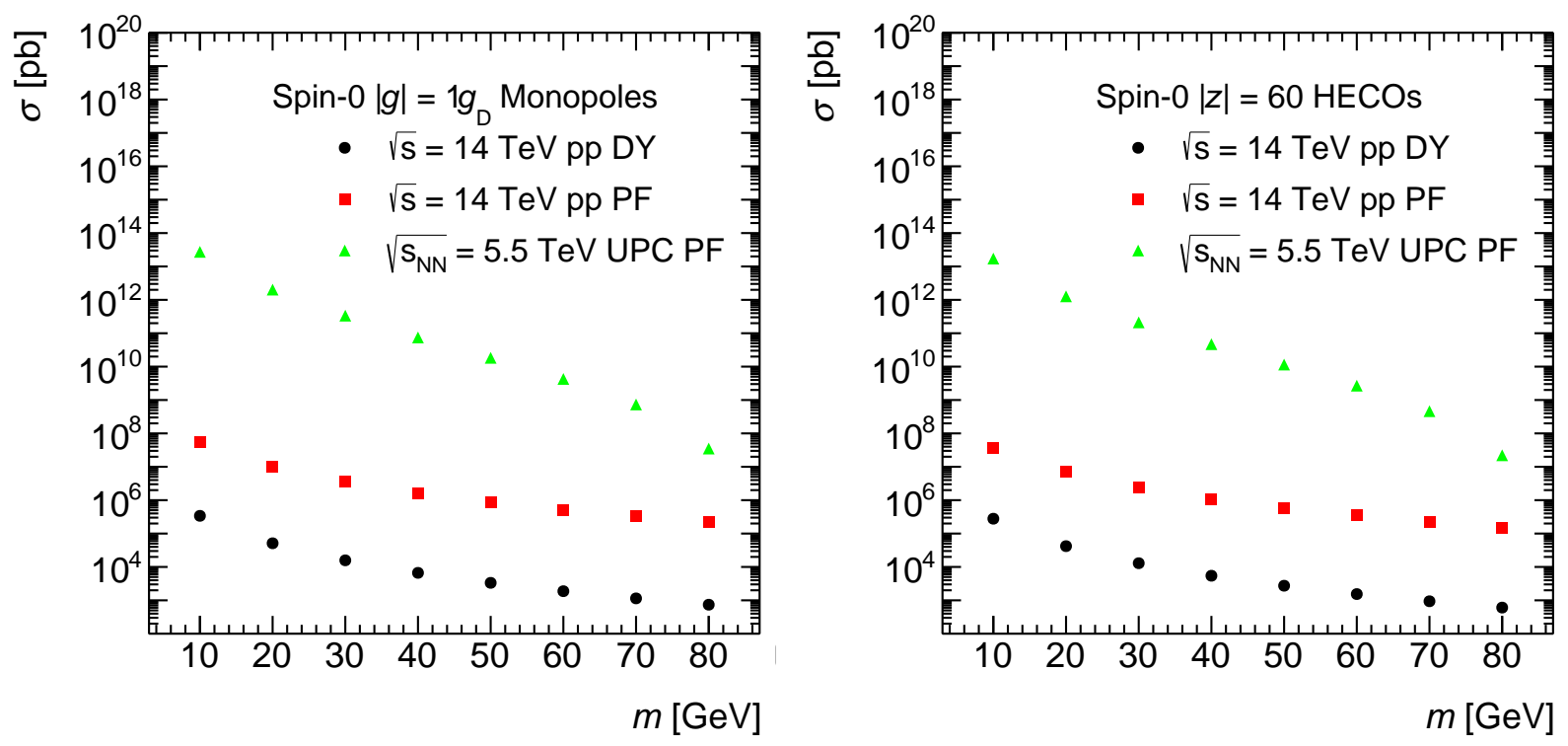

FIG. 10. Cross sections for spin-0 HIPs via the Drell-Yan (DY) and photon-fusion (PF) mechanisms in proton-proton (pp) collisions and via photon fusion (PF) in lead-lead ultraperipheral collisions (UPC).

In conclusion, we have studied the Drell-Yan and photon-fusion mechanisms of scalar and fermionic HIP production in $\sqrt{s_{\mathrm{NN}}}=5.5 \mathrm{TeV}$ heavy-ion ultraperipheral collisions and $\sqrt{s}=14 \mathrm{TeV}$ proton-proton collisions. The HIP kinematics are significantly more limited in the ultraperipheral collisions, hence, for comparison purposes their production in both collision types is examined in a mass range that is accessible in ultraperipheral collisions. In this mass range, their production in lead-lead ultraperipheral collision is significantly enhanced due to the large nuclear charge compared to the photon-fusion process in proton-proton collisions. Although this difference plays no role at the parton level on the matrix elements, it enhances the luminosity of the effective photon collisions. The spin- $\frac{1}{2}$ HECO production has a non-trivial interference effect between the $Z^{0}$-boson exchange and the photon exchange in the Drell-Yan mechanism, giving rise to a slightly smaller cross section than the exclusive photon-exchanged Drell-Yan mechanism. Nevertheless, the photon-fusion cross section exceeds that of Drell-Yan production in proton-proton collisions for the considered mass range as a consequence of its squared dependence of the HIP-photon coupling prevailing over the linear dependence of this coupling in the Drell-Yan cross section.

\section{ACKNOWLEDGMENTS}

We acknowledge Philippe Mermod's numerous contributions that led us to pursue this work. We thank Arka Santra, Ameir Shaa Bin Akber Ali, Stephanie Baines, Justin Kerr and Yury Smirnov for useful discussions on the modelling of the photon-fusion mechanism in $p p$ collisions. We thank Olivier Mattelaer for expert advice regarding implementing PDFs into MADGraPH5_aMC@NLO. We acknowledge the support of the Natural Sciences and Engineering Research 

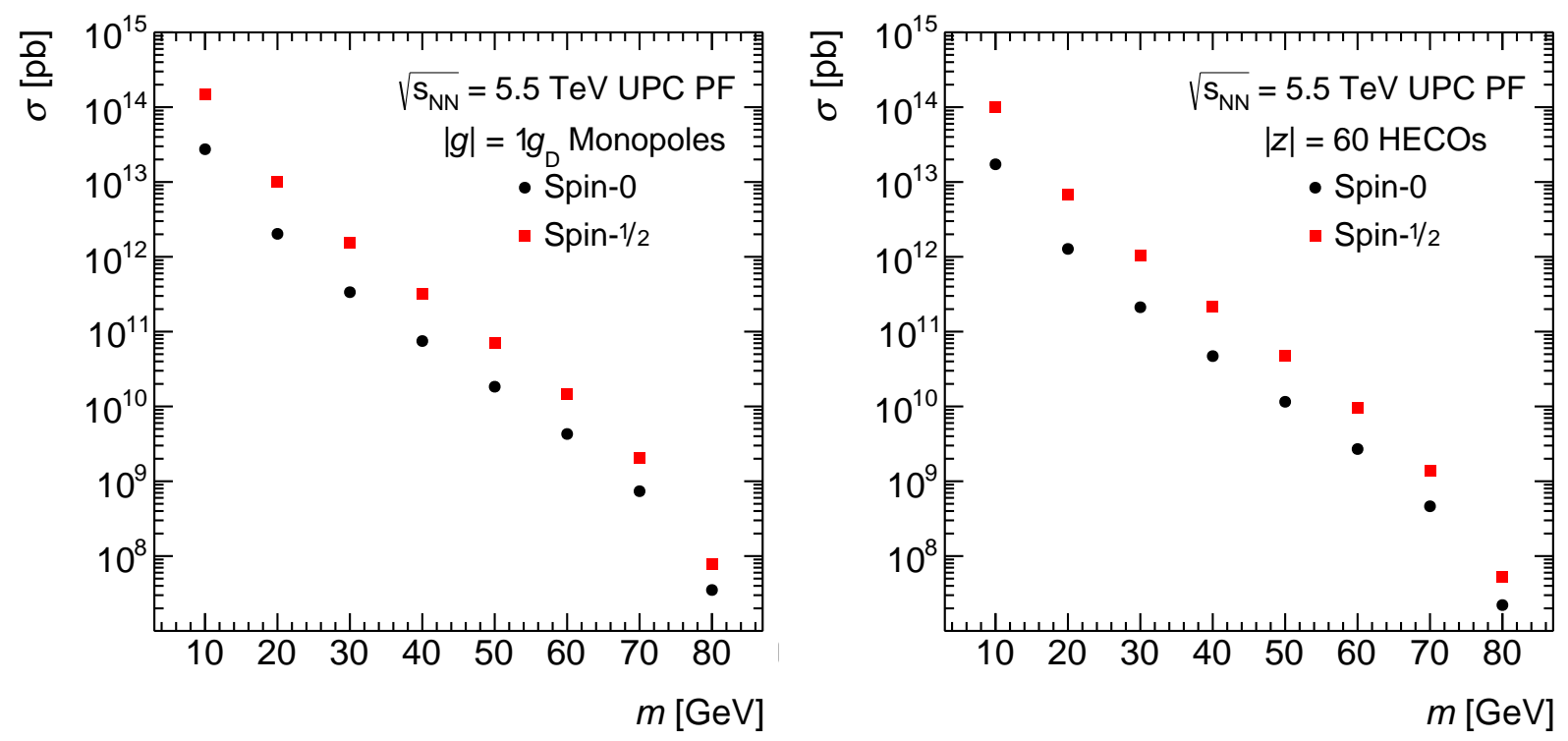

FIG. 11. Cross sections for spin-0 and spin- $\frac{1}{2}$ HIPs via photon fusion (PF) in lead-lead ultraperipheral collisions (UPC).
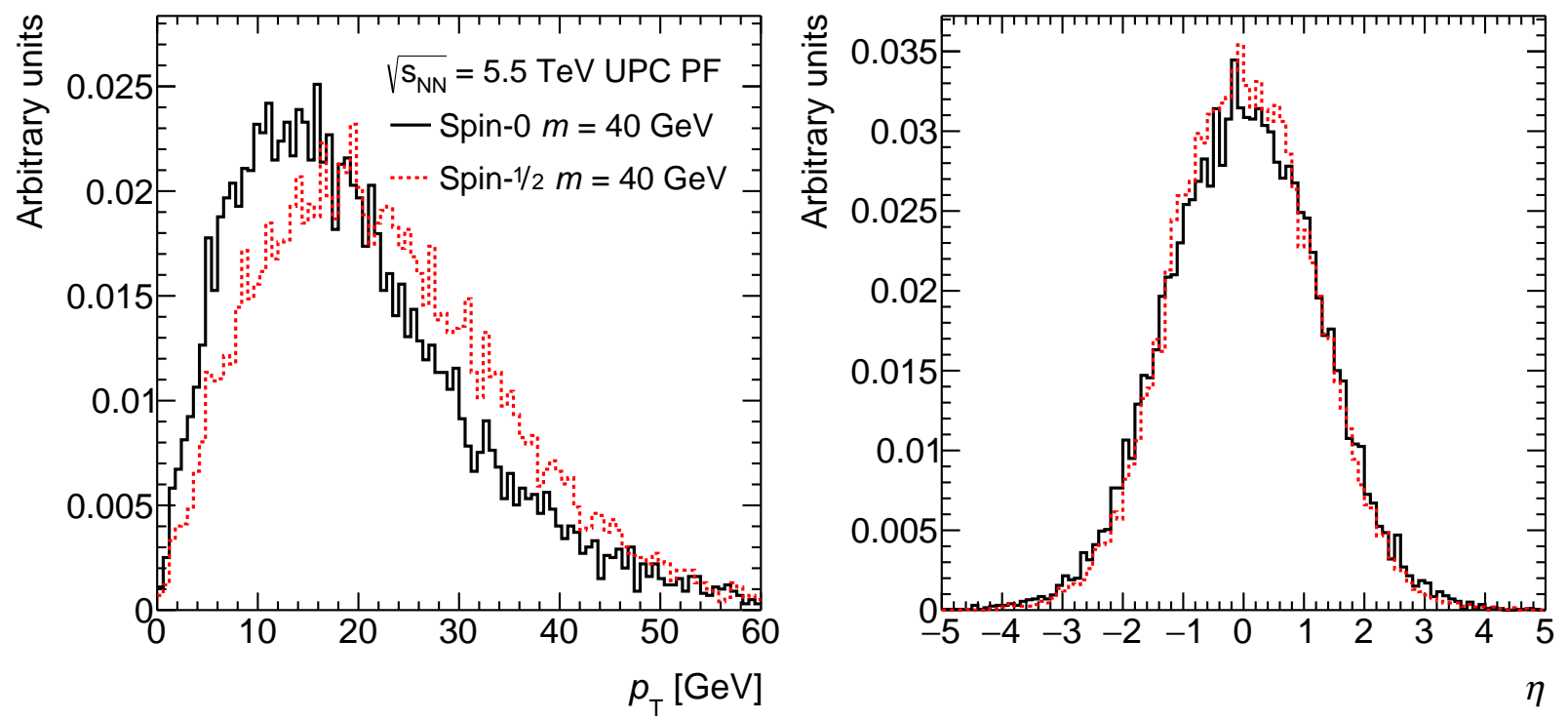

FIG. 12. Without loss of generality, $1 g_{\mathrm{D}}$ monopoles were chosen for the transverse momentum $p_{\mathrm{T}}$ and pseudorapidity $\eta$ of spin-0 and spin- $\frac{1}{2}$ HIPs with masses $40 \mathrm{GeV}$ produced in lead-lead ultraperipheral collisions (UPC).

Council of Canada (NSERC).

[1] P. A. M. Dirac, Quantised singularities in the electromagnetic field, Proc. R. Soc. A 133, 60 (1931).

[2] J. Preskill, Magnetic Monopoles, Ann. Rev. Nucl. Part. Sci. 34, 461 (1984)

[3] Y. M. Shnir, Magnetic Monopoles (Springer, New York, NY, 2005).

[4] K. A. Milton, Theoretical and experimental status of magnetic monopoles, Rept. Prog. Phys. 69, 1637 (2006), arXiv:hepex/0602040.

[5] E. J. Weinberg and P. Yi, Magnetic monopole dynamics, supersymmetry, and duality, Phys. Rept. 438, 65 (2007), arXiv:hep-th/0609055.

[6] A. Rajantie, Introduction to magnetic monopoles, Contemp. Phys. 53, 195 (2012), arXiv:1204.3077 [hep-th]. 

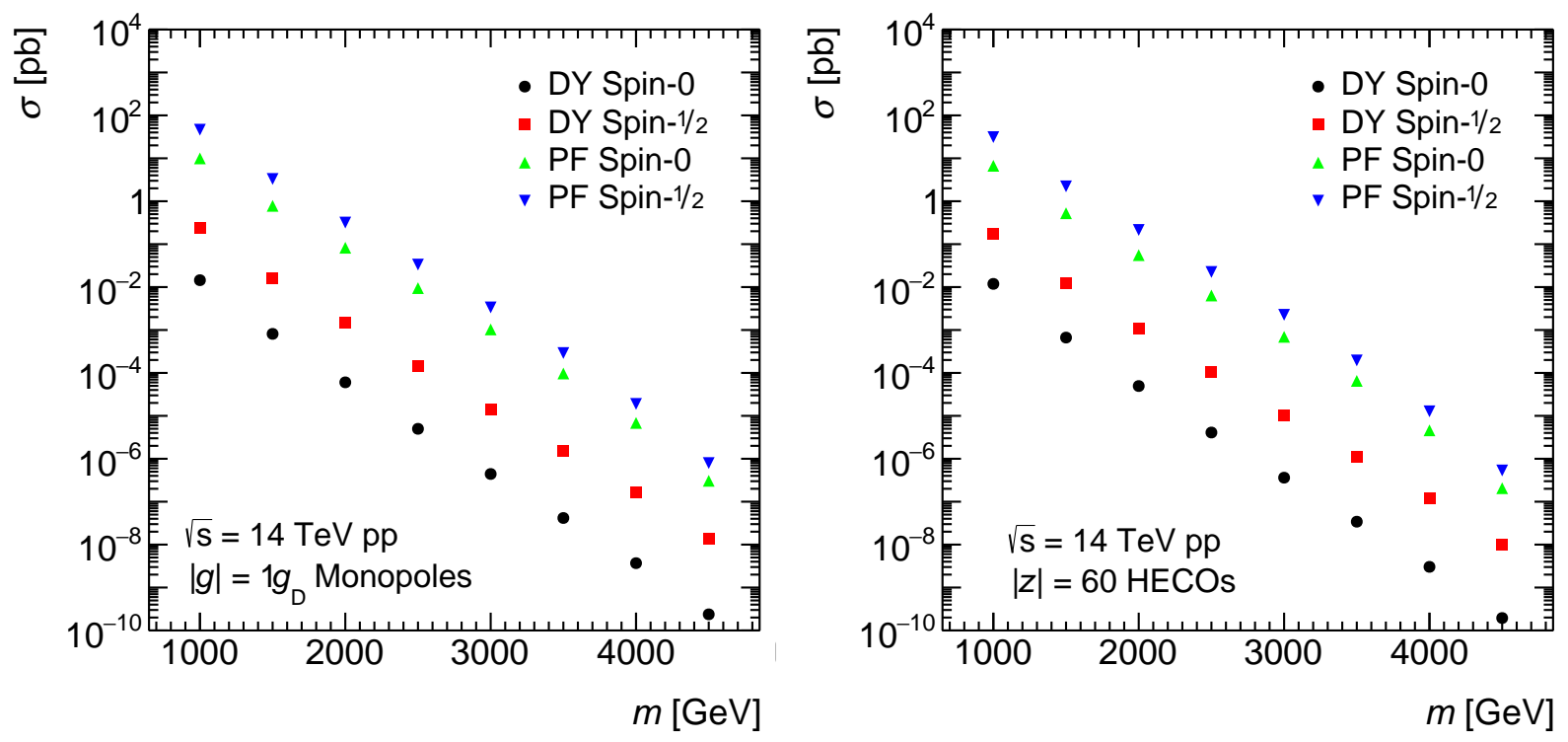

FIG. 13. Cross sections for spin-0 and spin- $\frac{1}{2}$ HIPs by Drell-Yan (DY) and photon fusion (PF) in proton-proton collisions.

[7] N. E. Mavromatos and V. A. Mitsou, Magnetic monopoles revisited: Models and searches at colliders and in the cosmos, Int. J. Mod. Phys. A 35, 2030012 (2020), arXiv:1305.1699 [hep-ph]

[8] G. 't Hooft, Magnetic monopoles in unified gauge theories, Nucl. Phys. B 79, 276 (1974)

[9] A. M. Polyakov, Particle spectrum in the quantum field theory, JETP Lett. 20, 194 (1974)

[10] T. W. Kephart, G. K. Leontaris, and Q. Shafi, Magnetic Monopoles and Free Fractionally Charged States at Accelerators and in Cosmic Rays, JHEP 10, 176, arXiv:1707.08067 [hep-ph].

[11] Y. M. Cho and D. Maison, Monopole configuration in Weinberg-Salam model, Phys. Lett. B 391, 360 (1997), arXiv:hepth/9601028

[12] K. Kimm, J. H. Yoon, and Y. M. Cho, Finite energy electroweak dyon, Eur. Phys. J. C 75, 67 (2015), arXiv:1305.1699 [hep-ph].

[13] J. Ellis, N. E. Mavromatos, and T. You, The price of an electroweak monopole, Phys. Lett. B 756, 29 (2016), arXiv:1602.01745 [hep-ph]

[14] S. Arunasalam and A. Kobakhidze, Electroweak monopoles and the electroweak phase transition, Eur. Phys. J. C 77, 444 (2017), arXiv:1702.04068 [hep-ph].

[15] J. Ellis, N. E. Mavromatos, and T. You, Light-by-Light Scattering Constraint on Born-Infeld Theory, Phys. Rev. Lett. 118, 261802 (2017), arXiv:1703.08450 [hep-ph]

[16] M. Arai, F. Blaschke, M. Eto, and N. Sakai, Localization of the Standard Model via the Higgs mechanism and a finite electroweak monopole from non-compact five dimensions, PTEP 2018, 10.1093/ptep/pty083 (2018), arXiv:1802.06649 [hep-ph].

[17] N. E. Mavromatos and S. Sarkar, Magnetic monopoles from global monopoles in the presence of a Kalb-Ramond Field, Phys. Rev. D 95, 104025 (2017), arXiv:1607.01315 [hep-th]

[18] N. E. Mavromatos and S. Sarkar, Regularized Kalb-Ramond magnetic monopole with finite energy, Phys. Rev. D 97, 125010 (2018), arXiv:1804.01702 [hep-th]

[19] P. Q. Hung, Topologically stable, finite-energy electroweak-scale monopoles, Nucl. Phys. B 962, 115278 (2021) arXiv:2003.02794 [hep-ph]

[20] J. Ellis, P. Q. Hung, and N. E. Mavromatos, An electroweak monopole, Dirac quantization and the weak mixing angle, Nucl. Phys. B 969, 115468 (2021), arXiv:2008.00464 [hep-ph].

[21] P. A. M. Dirac, The Theory of Magnetic Poles, Phys. Rev. 74, 817 (1948)

[22] S. P. Ahlen, Stopping Power Formula for Magnetic Monopoles, Phys. Rev. D 17, 229 (1978)

[23] S. P. Ahlen, Theoretical and experimental aspects of the energy loss of relativistic heavily ionizing particles, Rev. Mod. Phys. 52, 121 (1980), [Erratum: Rev.Mod.Phys. 52, 653-653 (1980)].

[24] S. p. Ahlen and K. Kinoshita, Calculation of the stopping power of very-low-velocity magnetic monopoles, Phys. Rev. D 26, 2347 (1982)

[25] G. Giacomelli and L. Patrizii, Magnetic monopole searches, ICTP Lect. Notes Ser. 14, 121 (2003), arXiv:hep-ex/0302011

[26] L. Patrizii and M. Spurio, Status of searches for magnetic monopoles, Annu. Rev. Nucl. Part. Sci. 65, 279 (2015) arXiv:1510.07125 [hep-ex].

[27] Y. Kurochkin, I. Satsunkevich, D. Shoukavy, N. Rusakovich, and Y. Kulchitsky, On production of magnetic monopoles via $\gamma \gamma$ fusion at high energy $p p$ collisions, Mod. Phys. Lett. A 21, 2873 (2006). 
[28] T. Dougall and S. D. Wick, Dirac magnetic monopole production from photon fusion in proton collisions, Eur. Phys. J. A 39, 213 (2009), arXiv:0706.1042 [hep-ph].

[29] L. N. Epele, H. Fanchiotti, C. A. García Canal, V. A. Mitsou, and V. Vento, Looking for magnetic monopoles at LHC with diphoton events, Euro. Phys. J. Plus 127, 60 (2012), arXiv:1205.6120 [hep-ph]

[30] S. Baines, N. E. Mavromatos, V. A. Mitsou, J. L. Pinfold, and A. Santra, Monopole production via photon fusion and Drell-Yan processes: MADGRAPH implementation and perturbativity via velocity-dependent coupling and magnetic moment as novel features, Eur. Phys. J. C 78, 966 (2018), [Erratum: Eur. Phys. J. C 79, 166 (2019)], arXiv:1808.08942 [hep-ph].

[31] ATLAS Collaboration, Search for Magnetic Monopoles in $\sqrt{s}=7 \mathrm{TeV} p p$ Collisions with the ATLAS Detector, Phys. Rev. Lett. 109, 261803 (2012), arXiv:1207.6411 [hep-ex].

[32] ATLAS Collaboration, Search for long-lived, multi-charged particles in $p p$ collisions at $\sqrt{s}=7$ TeV using the ATLAS detector, Phys. Lett. B 722, 305 (2013), arXiv:1301.5272 [hep-ex]

[33] ATLAS Collaboration, Search for magnetic monopoles and stable particles with high electric charges in 8 TeV $p p$ collisions with the ATLAS detector, Phys. Rev. D 93, 052009 (2016), arXiv:1509.08059 [hep-ex].

[34] ATLAS Collaboration, Search for Magnetic Monopoles and Stable High-Electric-Charge Objects in 13 TeV Proton-Proton Collisions with the ATLAS Detector, Phys. Rev. Lett. 124, 031802 (2020), arXiv:1905.10130 [hep-ex]

[35] MoEDAL Collaboration, Search for magnetic monopoles with the MoEDAL prototype trapping detector in 8 TeV protonproton collisions at the LHC, J. High Energy Phys. 2016 (08), 67, arXiv:1604.06645 [hep-ex]

[36] MoEDAL Collaboration, Search for Magnetic Monopoles with the MoEDAL Forward Trapping Detector in 13 TeV ProtonProton Collisions at the LHC, Phys. Rev. Lett. 118, 061801 (2017), arXiv:1611.06817 [hep-ex].

[37] MoEDAL Collaboration, Search for magnetic monopoles with the MoEDAL forward trapping detector in $2.11 \mathrm{fb}^{-1}$ of $13 \mathrm{TeV}$ proton-proton collisions at the LHC, Phys. Lett. B 782, 10.1016/j.physletb.2018.05.069 (2018), arXiv:1712.09849 [hep-ex]

[38] MoEDAL Collaboration, Magnetic Monopole Search with the Full MoEDAL Trapping Detector in 13 TeV pp Collisions Interpreted in Photon-Fusion and Drell-Yan Production, Phys. Rev. Lett. 123, 021802 (2019), arXiv:1903.08491 [hep-ex],

[39] B. Acharya, J. Alexandre, P. Benes, B. Bergmann, J. Bernabéu, A. Bevan, H. Branzas, P. Burian, M. Campbell, S. Cecchini, and et al., First search for dyons with the full moedal trapping detector in 13 tev $p p$ collisions, Phys. Rev. Lett. 126, 10.1103/physrevlett.126.071801 (2021), arXiv:2002.00861 [hep-ex]

[40] Y. D. He, Search for a Dirac Magnetic Monopole in High Energy Nucleus-Nucleus Collisions, Phys. Rev. Lett. 79, 3134 $(1997)$

[41] J. Schwinger, K. A. Milton, W.-Y. Tsai, L. L. DeRaad, and D. Clark, Nonrelativistic dyon-dyon scattering, Ann. Phys. 101, 451 (1976)

[42] J. T. Reis and W. K. Sauter, Production of magnetic monopoles and monopolium in peripheral collisions, Phys. Rev. D 96, 075031 (2017), arXiv:1707.04170 [hep-ph].

[43] J. Kang, P. Langacker, and B. D. Nelson, Theory and phenomenology of exotic isosinglet quarks and squarks, Phys. Rev. D 77, 035003 (2008), arXiv:0708.2701 [hep-ph].

[44] P. Langacker and G. Steigman, Requiem for a fractionally charged, massive particle, Phys. Rev. D 84, 065040 (2011), arXiv:1107.3131 [hep-ph]

[45] L. Roberts, Dirac magnetic-monopole pair production in relativistic nucleus-nucleus collisions, Il Nuovo Cimento A 92, 247 (1986)

[46] O. Gould and A. Rajantie, Thermal Schwinger pair production at arbitrary coupling, Phys. Rev. D 96, 076002 (2017), arXiv:1704.04801 [hep-th].

[47] O. Gould and A. Rajantie, Magnetic monopole mass bounds from heavy ion collisions and neutron stars, Phys. Rev. Lett. 119, 241601 (2017), arXiv:1705.07052 [hep-ph]

[48] O. Gould, D. L.-J. Ho, and A. Rajantie, Towards Schwinger production of magnetic monopoles in heavy-ion collisions, Phys. Rev. D 100, 015041 (2019), arXiv:1902.04388 [hep-th].

[49] A. Rajantie, Monopole-antimonopole pair production by magnetic fields, Phil. Trans. Roy. Soc. Lond. A 377, 20190333 (2019), arXiv:1907.05745 [hep-ph],

[50] D. L. J. Ho and A. Rajantie, Classical production of 't Hooft-Polyakov monopoles from magnetic fields, Phys. Rev. D 101, 055003 (2020), arXiv:1911.06088 [hep-th].

[51] D. L. J. Ho and A. Rajantie, Instanton solution for Schwinger production of 't Hooft-Polyakov monopoles, Phys. Rev. D 103, 115033 (2021), arXiv:2103.12799 [hep-th].

[52] O. Gould, D. L. J. Ho, and A. Rajantie, Schwinger pair production of magnetic monopoles: Momentum distribution for heavy-ion collisions, Phys. Rev. D 104, 015033 (2021), arXiv:2103.14454 [hep-ph].

[53] J. Schwinger, On Gauge Invariance and Vacuum Polarization, Phys. Rev. 82, 664 (1951)

[54] MoEDAL Collaboration, First experimental search for production of magnetic monopoles via the Schwinger mechanism, (2021), arXiv:2106.11933 [hep-ex].

[55] E. Fermi, On the theory of the impact between atoms and electrically charged particles, Z. Phys. 29, 315 (1924).

[56] C. F. Von Weizsäcker, Radiation emitted in collisions of very fast electrons, Z. Phys. 88, 612 (1934)

[57] E. J. Williams, Correlation of certain collision problems with radiation theory, Kong. Dan. Vid. Sel. Mat. Fys. Med. 13N4, 1 (1935).

[58] N. Bezginov, T. Valdez, M. Horbatsch, M. A., A. C. Vutha, and H. E. A., A measurement of the atomic hydrogen lamb shift and the proton charge radius, Science 365, 1007 (2019)

[59] J. Alwall, R. Frederix, S. Frixione, V. Hirschi, F. Maltoni, O. Mattelaer, H.-S. Shao, T. Stelzer, P. Torrielli, and M. Zaro, 
The automated computation of tree-level and next-to-leading order differential cross sections, and their matching to parton shower simulations, JHEP 07, 079, arXiv:1405.0301 [hep-ph].

[60] ATLAS Collaboration, Search for heavy long-lived multi-charged particles in proton-proton collisions at $\sqrt{s}=13$ TeV using the ATLAS detector, Phys. Rev. D 99, 052003 (2019), arXiv:1812.03673 [hep-ex]

[61] ATLAS Collaboration, Search for heavy long-lived multi-charged particles in $p p$ collisions at $\sqrt{s}=8$ TeV using the ATLAS detector, Eur. Phys. J. C 75, 362 (2015), arXiv:1504.04188 [hep-ex].

[62] ATLAS Collaboration, Search for long-lived, multi-charged particles in $p p$ collisions at $\sqrt{s}=7$ TeV using the ATLAS detector, Phys. Lett. B 722, 305 (2013), arXiv:1301.5272 [hep-ex].

[63] CMS Collaboration, Search for long-lived charged particles in proton-proton collisions at $\sqrt{s}=13$ TeV, Phys. Rev. D 94, 112004 (2016), arXiv:1609.08382 [hep-ex],

[64] CMS Collaboration, Searches for long-lived charged particles in $p p$ collisions at $\sqrt{s}=7$ and 8 TeV, JHEP 07, 122 , arXiv:1305.0491 [hep-ex].

[65] NNPDF Collaboration, Parton distributions with LHC data, Nucl. Phys. B 867, 244 (2013), arXiv:1207.1303 [hep-ph],

[66] A. Manohar, P. Nason, G. P. Salam, and G. Zanderighi, How Bright is the Proton? A Precise Determination of the Photon Parton Distribution Function, Phys. Rev. Lett. 117, 242002 (2016), arXiv:1607.04266 [hep-ph].

[67] V. Bertone, S. Carrazza, N. Hartland, and J. Rojo, Illuminating the photon content of the proton within a global PDF analysis, SciPost Phys. 5, 8 (2018), arXiv:1712.07053 [hep-ph].

[68] NNPDF Collaboration, Parton distributions from high-precision collider data, Eur. Phys. J. C 77, 663 (2017), arXiv:1706.00428 [hep-ph]

[69] NNPDF Collaboration, Parton distributions with QED corrections, Nucl. Phys. B 877, 290 (2013), arXiv:1308.0598 [hep$\mathrm{ph}]$.

[70] J. D. Jackson, Classical electrodynamics; 2nd ed. (Wiley, New York, NY, 1975) p. 727.

[71] M. Abramowitz and I. A. Stegun, Handbook of Mathematical Functions, With Formulas, Graphs, and Mathematical Tables (Dover Publications, Inc., USA, 1974) pp. 378-379. 